\title{
Thoughts on Addiction Prevention
}

\author{
J. B. Moores \\ Rockaway Place, Lexington, KY, USA \\ Email: br00ke127@aol.com
}

How to cite this paper: Moores, J. B. (2019). Thoughts on Addiction Prevention. Psychology, 10, 88-95.

https://doi.org/10.4236/psych.2019.101007

Received: December 24, 2018

Accepted: January 20, 2019

Published: January 23, 2019

Copyright $\odot 2019$ by author(s) and Scientific Research Publishing Inc. This work is licensed under the Creative Commons Attribution International License (CC BY 4.0).

http://creativecommons.org/licenses/by/4.0/

(c) (i) Open Access

\begin{abstract}
All drugs of addiction produce a positive reward with efficacy and once the efficacy tapers off so do the effects from the drug. Despite the consequences that can occur from an addicting behavior, individuals like how the reward made them feel. Therefore, repeating the behavior over and over creates a cycle of addiction. Equally important, is the social concern from the mortality rate statistics from drug addiction listed from most to least overdose: opioid, cocaine, and benzodiazepines. According to the Centers for Disease Control and Prevention statistics and in Figure 1. "More than 72,000 Americans died from drug overdose in 2017; 49,068 involve opioid overdose, 14,556 involve cocaine overdose; and 10,684 involve benzodiazepine overdose" (National Institute on Drug Abuse, 2018). Within this commentary article, there will be suggestive research for an optimistic outlook on novel ways to approach our nation's crisis. In addition, there will be some examples of the most common prescription drug types describing their mechanism of action. Finally, discussing the stages of drug addiction to reveal where relapse occurs so there can be better monitoring and documentation while help aiding with some of the Health and Human Services 5-Point Strategy, "Better addition prevention, treatment, and recovery services, better data, better pain management, better targeting of overdose reversing drugs, and better research" (U.S. Department of Health and Human Services, 2018).
\end{abstract}

\section{Keywords}

Chronic Pain, Relapse, Misuse, Abuse, Addiction, Medically Necessary

\section{Introduction}

Everybody has an addictive behavior whether the addictive behavior may be but not limited to: eating, smoking, sex, gambling, drinking, or drugs. Accompanied with an addictive behavior is a neurotransmitter in the brain called, "dopamine". "Dopamine helps regulate movement, attention, learning, and emotional responses. 


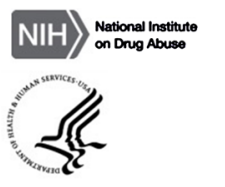

\section{National Overdose Deaths}

Number of Deaths Involving Opioids
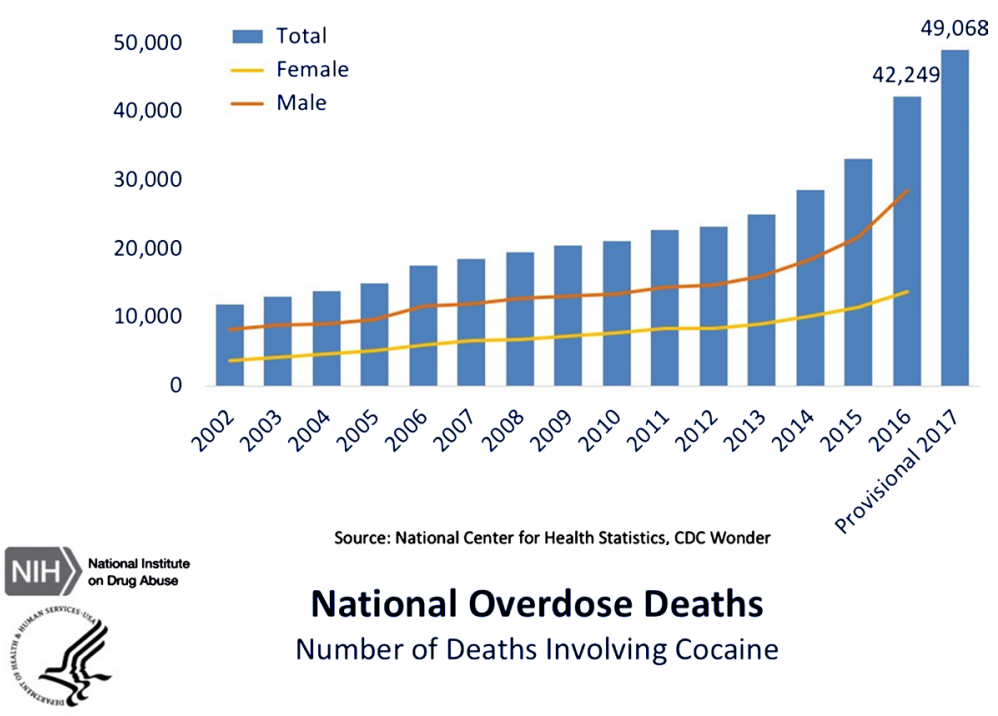

National Overdose Deaths

Number of Deaths Involving Cocaine

2

$\begin{array}{ll}16,000 & - \text { Total } \\ 14,000 & \text { - Female } \\ 12,000 & \text { - Male }\end{array}$

12,000

10,000

8000

6000

4000

2000

0

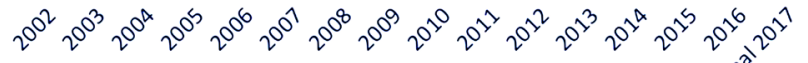

$\mathrm{NIH}>\substack{\text { Nabional Insturte } \\ \text { on Drug Abuse }}$

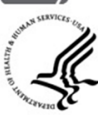

Source: National Center for Health Statistics, CDC Wonder

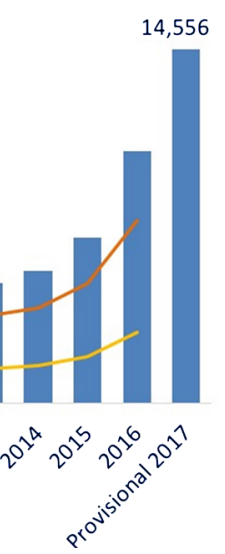

National Overdose Deaths

Number of Deaths Involving Benzodiazepines

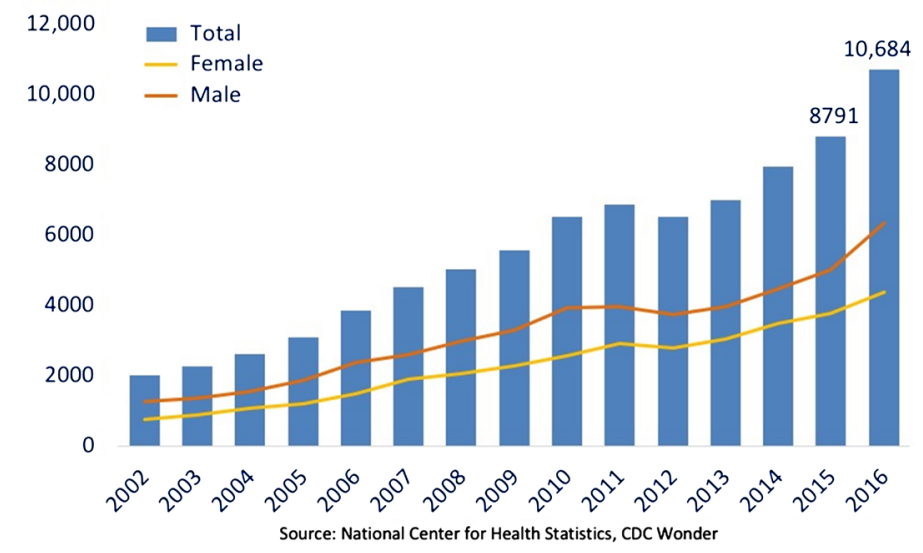

Figure 1. Overdose death rates (Source:

https://www.drugabuse.gov/related-topics/trends-statistics/overdose-death-rates). 
It also enables us not only to see rewards, but to take action to move toward them. Since dopamine contributes to feelings of pleasures and satisfaction as part of the reward system, the neurotransmitter also plays a part in addiction" (Psychology Today, 2018). Moreover, drug addiction is one addictive behavior that has impacted our nation severely from the use, abuse, and misuse of opioids. On October 26, 2017 the Secretary of the Department of Health and Human Services, Eric Hargan declared, "As a result of the consequences of the opioid crisis affecting our Nation, on this date and after consultation with public health officials as necessary, I, Eric D. Hargan, Acting Secretary of Health and Human Services, pursuant to the authority vested in me under section 319 of the Public Health Service Act, do herby determine that a public health emergency exist nationwide" (Department of Health \& Human Service, 2017).

\section{Stages of Addiction}

In view of addictive behavior, there are stages that can lead up to addiction which is a habit, and it takes a habit to break a habit. According to the Food Drug and Administration (FDA) warning section on the drug packet insert for prescription drugs of potential addiction, "Addiction can occur at recommended dosages and if the drug is misused or abused". The first stage of addiction is use. Use is where the drug begins its route of administration for the first time, along with duration and frequency that is medically necessary to accomplish a result. This is also the stage where the feelings of the effects of the drug are discovered. Given this point, the CDC guidelines for prescribing opioids for chronic pain should be mandatory implemented and used as guidance for better prescription management. According the CDC guidelines for prescribing opioids for chronic pain, the first step in determining when a drug should be received states, "Nonpharmacologic therapy and nonopioid pharmacologic therapy are preferred for chronic pain. Clinicians should consider opioid therapy only if expected benefits for both pain and function are anticipated to outweigh risks to the patient. If opioids are used, they should be combined with nonpharmacologic therapy and nonopoid pharmacologic therapy as appropriate" (Centers for Disease Control and Prevention, 2017). The second stage is continued use of the drug or substance and remembering how the drug made the individual feel. Equally important, the continued use of a drug can also alter the chemical function of your brain. "Acutely, the drugs are rewarding, and this effect promotes repeated use" (Julien et al., 2011: p. 569). Leading to the next stage, tolerance, where your body requires more of the drug from repeated use in order to achieve the same feeling from first use. "Drug tolerance is defined as a state of progressively decreasing responsiveness to a drug. A person who develops tolerance requires a larger dose of the drug to achieve the effect originally obtained by a smaller dose" (Julien et al., 2011: p. 33). Notably, there are several mechanisms that help contribute to drug tolerance. "At least three mechanisms are involved in the development of drug tolerance-two are pharmacological mechanisms; one is a behavioral me- 
chanisms. Metabolic tolerance, the first tolerance of the two classically described types of pharmacologic tolerance, more enzyme is available to metabolize a drug and, as a result, more drug must be administered to maintain the same level of drug in the body. Cellular-adaptive, or pharmacodynamics, tolerance is the second type of pharmacological tolerance. Receptors in the brain adapt to the continued presence of the drug, with neurons adapting to excess drug either by reducing the number of receptors available to the drug or by reducing their sensitivity to the drug" (Julien et al., 2011: p. 33). Next is behavior conditioning processes, "Can be demonstrated when a drug is administered in the context of usual pre-drug cues but not in the context of alternative cues" (Julien et al., 2011: p. 33). For instance, the illustration proposed by, "Poulos and Cappell homeostatic theory when testing analgesia and environment. They found that, with morphine analgesia, testing in an environment in which tolerance had developed affected the manifestation of tolerance, and environmental cue could maintain the tolerance" (Julien et al., 2011: p. 33). Finally, environmental cues that create a behavioral stimulus with administering drugs. "The environmental cues routinely paired with drug administration will become conditioned stimuli that elicit a conditioned response that is opposite in direction to or compensation for the direct effects of the drug" (Julien et al., 2011: p. 33). The fourth stage is Physical dependence; this is where your body physically depends on the drug to avoid withdrawal. "A person who is physically dependent needs the drug to avoid the withdrawal symptoms that occur if the drug is not taken. The state is revealed by withdrawing the drug and noting the occurrence of physical and/or psychological changes (withdrawal symptoms)" (Julien et al., 2011: p. 33). Also, this is the same stage where relapse occurs from the cave and withdrawal of a drug. "If the drug is withdrawn, similar unpleasant emotional reactions and changes in the brain will occur, regardless of the specific drug; it is these effects that are responsible for craving and relapse" (Julien et al., 2011: p. 569). Finally, addiction has occurred from abuse and misuse of a drug that has chemical altered the brain. "The addicted individual experiences urges to use the drug again and is at risk of relapsing. The development of such cravings indicates that something in the brain has changed as a result of long-term drug use; some process of neuroadaptation has occurred" (Julien et al., 2011: p. 569).

\section{Pharmacokinetics}

\subsection{Analgesics}

Pain is an unpleasant physical or emotional stimulus when encountered by an experience. "Pain can be defined as a highly undesirable and unpleasant sensory and emotional experience often associated with actual or potential tissue damage" (Julien et al., 2011: p. 315). The two types of pain that can be experienced are acute pain and chronic pain. "Acute pain is biologically desirable because it functions as a warning system against real or potential damage to the body. Chronic pain, however, serves no useful purpose, causes suffering, limits activi- 
ties of daily living, and increases the costs of health care and disability" (Julien et al., 2011: p. 315). Additionally, "Chronic pain sets up ongoing spinal processes of neuroadaptation with associated physiological changes in spinal circuitry. Chronic pain is thought to be poorly responsive to opioids but quite responsive to anticonvulsants such as gabapentin. For this reason, treatment of chronic pain with only opioid therapy is usually ill-advised because efficacy is limited and the risk of developing drug dependence is high" (Julien et al., 2011: p. 319). Some of the most commonly prescribed opioids include, "Lortab, Percocet, Morphine, Codeine, and Fentanyl" (National Institute on Drug Abuse, 2018). All of these opioids have a mechanism of action in common with the dopamine pathway in the brain. "The system consists of one of the major brain pathways for dopamine, called the mesolimblic dopamine pathway" (Julien et al., 2011: pp. 569-570). Additionally, "Opioids exert their pharmacological actions through three opioid receptors, mu, delta, and kappa" (Science News, 2007). However, the commonly prescribed opioids share the mu opioid receptors. For example, "Percocet is a full opioid agonist with relative selectivity for the mu-opioid receptor, although it can interact with other opioid receptors at higher doses" (FDA packet insert).

\subsection{Cocaine}

Cocaine is an illegal powerful psychostimulant that affects the dopamine reward circuit in the brain. Even though cocaine has a past usage for different treatment, such as, local anesthetic, fatigue, and help with opioid addiction. Cocaine is an illegal second class drug from overdose resulting in demise. However, cocaine's mechanism of action is, "Currently, most focus is on cocaine's blockade of presynaptic transporter for dopamine as being crucial to its behavior reinforcing and psycostimuliation properties" (Julien et al., 2011: p. 403). As dopamine re uptake is blocked in the presynaptic it allows dopamine to "hang out" much longer to achieve the euphoric effects. Comparably, there are controlled prescription medications mechanism of action that works similar to cocaine. Such as, amphetamine and methamphetamines, which are prescription medications used to treat mostly for a brain disorder, attention-deficit/hyperactivity disorder (ADHD) in children. "For instance, Adderall is a commonly prescribed amphetamines and on the drug pack insert for Adderall it states, Amphetamines is thought to block the reuptake of norepinephrine and dopamine into the presynaptic neuron and increase the release of these monoamines into the extramural space" (FDA, 2007). Regarding this, "The behavioral stimulation and increased psychomotor activity appear to follow from the resulting stimulation of the dopamine receptors in the mesolimbic system, including the nucleus accumbens" (Julien et al., 2011: pp. 415-416). Likewise, a commonly prescribe methamphetamine is Focalin and, "thought to block the reuptake of norepinephrine and dopamine into the presynaptic neuron and increase the release of these monoamines into the extra neuronal space" (FDA drug insert packet). Not to mention, "The effects of methamphetamine closely resemble the effects produced by co- 
caine. Both drugs are potent psychomotor stimulants and positive reinforces" (Julien et al., 2011: p. 420).

\subsection{Benzodiazepines}

There are several benzodiazepines that are used to treat a variety of diagnoses such as, anxiety, insomnia, seizures, and alcohol withdrawal. Some of the most commonly prescribed benzodiazepines are, "Valium, Xanax, Klonopin, and Ativan" (Julien et al., 2011: p. 238). Benzodiazepines bind to a different receptor in the brain from analgesic and psychostimulants. "Benzodiazepines exert their anxiolytic properties by acting on GABA neurons at the limbic centers" (Julien et al., 2011: p. 239). In addition, to GABA neurotransmitter, "Benzodiazepines serve as agonist by binding to a site near GABA-binding site and by facilitating the action of GABA in increasing the flow of chloride ions into the neuron. Because chloride ions are negative, their inward flow hyperpolarizes the neuron and inhibits neuronal function. This action underlies the use of benzodiazepines as sedative, antianxiety, amnestic, and antiepileptic agents" (Julien et al., 2011: pp. 41-42).

\section{Suggestions for Future Research}

Several suggestions for future research to help with our nation's crisis and decrease the amount of opioid prescriptions and other addicting drugs. First, mandatory implementation of the CDC guidelines for prescribing opioids for chronic pain to help aid the provider in deciding if the prescription management is medically necessary and/or if the risk outweigh the benefits. According to Centers for Medicare \& Medicaid Services (CMS) their term medically necessary is defined as, "Services or supplies that: are proper and needed for the diagnosis or treatment of your medical condition, are provided for the diagnosis, direct care, and treatment of your medical condition, meet the standards of good medical practice in the local area, and aren't mainly for the convenience of you or your doctor" (Centers for Medicare \& Medicaid Services, 2006). Also, there should be mandatory critical key elements in the narcotic agreement form making the consequences well publicized for abuse and misuse and defining the terms for the patient and provider for prescription management. Additionally, if there are any controlled prescriptions that are picked up at a pharmacy prior to a procedure. There should be a pill count and if there are any remaining pills from the procedure the provider's office should be responsible for destroying. Another suggestion for future research is to examine the drug class, controlled and non-controlled as well as drug type to see if a relationship exist for potential addiction. Such as, by making a chart for illustration purposes for prescription drug comparison awareness of the potential addiction with future prescription medications approved by the Food Drug and Administration (FDA). For example, amphetamine and methamphetamines mechanism of action is very similar to cocaine and with supporting data that displays a correlation with long term use of amphetamine and methamphetamines in children are more likely to use cocaine 


\begin{tabular}{ccc}
\hline & Presented & Removed \\
\hline Increase Response Rate & Positive Reinforcement & Negative Reinforcement \\
Decrease Response Rate & Positive Punishment & Negative Punishment \\
\hline
\end{tabular}

Figure 2. Response consequence contingences (Morgan, 2002).

later in life. "Many cocaine abusers seeking treatment have a history of childhood attention deficit/hyperactive disorder (ADHD), and approximately 15 percent of cocaine abusers seeking treatment may have adult ADHD" (Julien et al., 2011: p. 412). While taking into consideration the rise of ADHD diagnoses has increased in children from 2003 to 2011. "Health care providers who care for children with attention-deficit/hyperactivity disorder (ADHD) and public health practitioners should be aware that an estimated two million more U.S. children were reported by their parents to be diagnosed by a health care provider with ADHD and a million more were reported to be taking medication for ADHD in 2011, compared to 2003. These health professionals should also be aware of the changing patterns of ADHD in the United States" (Centers for Disease Control and Prevention, 2018). Current data compared to 2011 to 2016 from the CDC shows data is almost identical with, "6.1 million children aged 2 - 17 years living in the U.S. had been diagnosed with attention-deficit/hyperactivity disorder (ADHD), which is similar to the previous estimates" (Centers for Disease Control and Prevention, 2018). Also, primary care providers should continue their ADHD forms for screening just like the MCHAT screening for autism. If the screening is positive, then refer to a behavior specialist for further testing. Another example, includes the commonly prescribed opioids, found through the same dopaminergic pathway, thus causing such addiction leading to overdose. Instead of making newer opioid medications to treat chronic pain, maybe new approved prescription medication should focus more on type and class of anticonvulsants. Furthermore, since physical dependence is the stage of addiction where relapse occurs there should be better documentation and monitoring at withdrawal since caving and relapse occur here. Also, ICD-10 codes of selection from chapter 5 in the 2019 ICD-10 CM Expert that should be closely monitored for data when coded to specificity, "dependence, drug by type, with withdrawal" (AAPC, 2018: pp. 539-567). Another suggestion for future research is for public schools to consider incorporating life skills and responsibility into the curriculum to teach students how to be a responsible adult while being responsible for their actions. Additionally, teaching/showing children how to do life skills, that will enable them to deal with demands and challenges throughout life. Lastly, for individuals seeking help or willing to get help for their drug addiction, this should be done through drug court while using the response consequence contingencies chart. For this reason, if an individual can be drug free and not relapse, then any felony drug related charges should be expunged, in exchange of helping the individual regain their dignity and hope. Please refer to Figure 2. 


\section{Conflicts of Interest}

The author declares no conflicts of interest regarding the publication of this paper.

\section{References}

AAPC (2018). ICD-10 2019 CM Expert for Providers and Facilities. (pp. 539-567).

Centers for Disease Control and Prevention (2017). CDC Guidelines for Prescribing Opioids for Chronic Pain.

https://www.cdc.gov/drugoverdose/prescribing/guideline.html

Centers for Disease Control and Prevention (2018). Attention-Deficit/Hyperactivity Dis$\operatorname{order}(A D H D)$.

https://www.cdc.gov/ncbddd/adhd/features/key-findings-adhd72013.html

Centers for Medicare \& Medicaid Services (2006). Glossary. https://www.cms.gov/apps/glossary/default.asp?Letter=M\&Language=English

Department of Health \& Human Services (2017). Determination That a Public Health Emergency Exists.

https://www.hhs.gov/sites/default/files/opioid\%20PHE\%20Declaration-no-sig.pdf

Food Drug Administration (2007). Adderall (C11). https://www.accessdata.fda.gov/drugsatfda_docs/label/2007/011522s040lbl.pdf

Julien, R. M., Advokat, C. D, \& Comaty, J. E. (2011). A Primer of Drug Action (12th ed., pp. 33, 41-42, 238-239, 315, 319, 403, 412, 415-416, 420, 569-570). Long Grove, IL: Waveland Press, Inc.

Morgan, D. L. (2002). Essentials of Learning and Cognition (p. 111). New York, NY: Worth Publishers.

National Institute on Drug Abuse (2018). Prescription Opioids. https://www.drugabuse.gov/publications/drugfacts/prescription-opioids

Psychology Today (2018). Dopamine. https://www.psychologytoday.com/us/basics/dopamine

Science News (2007). How Does the Opioid System Control Pain, Reward, and Addictive Behavior? https://www.sciencedaily.com/releases/2007/10/071014163647.htm

U.S. Department of Health and Human Services (2018). 5-Point Strategy to Combat the Opioid Crisis.

https://www.hhs.gov/opioids/about-the-epidemic/hhs-response/index.html 